\title{
SOME RESULTS ON HOMOCLINIC AND HETEROCLINIC CONNECTIONS IN PLANAR SYSTEMS
}

\author{
A. GASULL*, H. GIACOMINI, AND J. TORREGROSA*
}

\begin{abstract}
Consider a family of planar systems depending on two parameters $(n, b)$ and having at most one limit cycle. Assume that the limit cycle disappears at some homoclinic (or heteroclinic) connection when $\Phi(n, b)=0$. We present a method that allows to obtain a sequence of explicit algebraic lower and upper bounds for the bifurcation set $\Phi(n, b)=0$. The method is applied to two quadratic families, one of them is the well-known Bogdanov-Takens system. One of the results that we obtain for this system is the bifurcation curve for small values of $n$, given by $b=\frac{5}{7} n^{1 / 2}+\frac{72}{2401} n-\frac{30024}{45294865} n^{3 / 2}-\frac{2352961656}{11108339166925} n^{2}+O\left(n^{5 / 2}\right)$. We obtain the new three terms from purely algebraic calculations, without evaluating Melnikov functions.
\end{abstract}

\section{INTRODUCTION}

Consider a smooth family of planar differential equations $(\dot{x}, \dot{y})=(P(x, y ; n, b)$, $Q(x, y ; n, b)),(n, b) \in \mathbb{R}^{2}$, for which the existence of at most one limit cycle is already known and moreover all the bifurcations occurring in the family are well understood. For this family we could say that the Qualitative theory of ordinary differential equations has achieved all its goals and all the job is done. Nevertheless, from an analytic and quantitative point of view there remains a crucial question: to determine the bifurcation curves in the bifurcation diagram of the family.

Some of these bifurcation curves are not difficult to find. This is the case for instance of the curves that control the changes in the behavior of the flow near the critical points. These bifurcation curves correspond to local phenomena and so, in principle, are easier to study. A paradigmatic example of this kind is the bifurcation curve associated to the birth of a limit cycle due to an AndronovHopf bifurcation. The curves governing global phenomena are in general much more difficult to determine. This is, for instance, the case of the appearance of homoclinic or heteroclinic connections.

We propose a method that, in some cases, allows to obtain explicit lower and upper bounds in the parameter plane for the location of the bifurcation curves associated to these global phenomena. As illustration, we will apply it to two 2parametric families of quadratic systems having at most one limit cycle. Since our examples are polynomial, we will consider their phase portraits on the Poincaré

2000 Mathematics Subject Classification. Primary 34C23; Secondary 34C25, 34C37, 37C27.

Key words and phrases. polynomial planar system, limit cycle, bifurcation, homoclinic and heteroclinic orbits, Bogdanov-Takens system.

*Partially supported by a MCYT/FEDER grant number MTM2008-03437 and by a CIRIT grant number 2009SGR 410. 
sphere, see for instance [11, Chap. 5] or [14, Chap. 3.10], and the homoclinic or heteroclinic connections will also take into account the critical points at infinity.

We start by giving a description of the method for finding the relation between the parameters $(n, b)$ for which this homoclinic or heteroclinic connection exists. As a first step we consider an algebraic curve $\mathcal{C}=\left\{(x, y) \in \mathbb{R}^{2}: C(x, y)=0\right\}$, as general as possible, but having some of the geometrical properties of the phase portrait of the system. For instance, it has to pass through the two critical points of the vector field that are connected by the heteroclinic orbit; if the critical point is a saddle it has to be tangent to one of its separatrices; if the critical point is at infinity, it has to have a branch going to infinity in the direction corresponding to this point, etc.

The second step consists in using all the free parameters in $\mathcal{C}$ to impose that, near the critical points, the curve be as close as possible to the separatrix connecting orbit. In most cases this can be done by finding a local analytical expression of this separatrix in the neighborhood of each critical point. There are several possibilities to impose that $\mathcal{C}$ be as close as possible to the separatrix. If the separatrix is a heteroclinic orbit we can fix all the free parameters of $\mathcal{C}$ imposing this condition only in the neighborhood of one of the critical points or in the neigbourhood of both critical points at the same time. If the separatrix is a homoclinic orbit we can impose the condition only on one of the branches of the orbit that arrives to the critical point or on the two at the same time. In the two families of quadratic systems studied in the next sections all these possibilities will be explored.

Once all the parameters of the curve $\mathcal{C}$ are fixed, we impose one more level of closeness between both objects (the connecting orbit and the algebraic curve) by forcing a relation among the parameters $(n, b) \in \mathbb{R}^{2}$. This procedure gives a curve $\mathcal{B}=\left\{(n, b) \in \mathbb{R}^{2}: \Phi(n, b)=0\right\}$ in the bifurcation space. In fact, up to this point the method proposed is essentially the one followed in [2] to obtain analytic approximations to separatrices for some two-point boundary value problems.

The last step is our main contribution and is the one that leads to explicit algebraic upper and lower bounds of the bifurcation curve associated to the existence of connections between the two critical points. This is the most difficult and computationally involved part of the method. We have to prove that there are parameters $(n, b)$ near the curve $\mathcal{B}$ such that the corresponding curves $\mathcal{C}$ are without contact for the flow of the vector field and moreover that there are values for which the flow crosses the curve in one sense and values for which the crossing is in the opposite direction. This will be clearer in our applications.

Notice that the aim of our work is in the spirit of what Coppel proposed in his well-known paper [5]: "Ideally one might hope to characterize the phase portraits of quadratic systems by means of algebraic inequalities on the coefficients", taking into account the results of [7] where the authors proved that there are bifurcation curves in quadratic systems which are neither algebraic nor analytic. Since there is no hope to find analytic or algebraic expressions of the bifurcation curves, we try to sandwich them between two algebraic curves.

We present below our main results. The first one deals with a family of quadratic systems already studied in [15] and the second one with the Bogdanov-Takens system. For both systems there is at most one (hyperbolic) limit cycle. The value 
$b=b^{*}(n)$ corresponds, for each $n$, to the value where this limit cycle, which is born in an Andronov-Hopf bifurcation, disappears giving rise to a heteroclinic or homoclinic connection, respectively, see more details in the next sections.

Theorem 1.1. Let $b=b^{*}(n)$ be the bifurcation curve corresponding to the heteroclinic connection for the quadratic system

$$
\left\{\begin{array}{l}
\dot{x}=P(x, y)=y, \\
\dot{y}=Q(x, y)=-x+b y+x y-n y^{2},
\end{array}\right.
$$

with $b>0, n>0$. Then:

(i) For $n>14$,

$$
\left|\frac{1}{4 n}-b^{*}(n)\right|<\frac{1}{8 n^{3}} .
$$

(ii) For $n>5$,

$$
\left|\frac{1}{4 n}-\frac{1}{64 n^{3}}-b^{*}(n)\right|<\frac{1}{2 n^{5}} .
$$

(iii) For $n>26$,

$$
\left|\frac{1}{4 n}-\frac{1}{64 n^{3}}-\frac{5}{512 n^{5}}-b^{*}(n)\right|<\frac{1}{8 n^{7}} .
$$

Theorem 1.2. Let $b=b^{*}(n)$ be the bifurcation curve corresponding to the saddle loop homoclinic connection for the Bogdanov-Takens system

$$
\left\{\begin{array}{l}
\dot{x}=y \\
\dot{y}=-n+b y+x^{2}+x y
\end{array}\right.
$$

with $n>0$. Then $b^{*}(n)=H(\sqrt{n})$, where $H$ is an analytic function. Moreover,

(i) For $0 \leq n \leq 1 / 40$,

$$
\left|\frac{5}{7} \sqrt{n}+\frac{72}{2401} n-b^{*}(n)\right|<\frac{11}{4} n^{5 / 4} .
$$

(ii) For $n$ small enough,

$$
b^{*}(n)=\frac{5}{7} n^{1 / 2}+\frac{72}{2401} n-\frac{30024}{45294865} n^{3 / 2}-\frac{2352961656}{11108339166925} n^{2}+O\left(n^{5 / 2}\right) .
$$

The fact that $b^{*}(n)=H(\sqrt{n})$, where $H$ is an analytic function, is an easy consequence of some results of [13], see Corollary 3.5. Our main contributions are items (i) and (ii). Notice that they improve the well-known local knowledge of the function $b=b^{*}(n)=\frac{5}{7} \sqrt{n}+O(n)$, proved for instance in [8] by using a blowing-up process and scaling of the time and the computation of a Melnikov function. In Section 3.5 we compare them with other results of Perko obtained in the mentioned paper [13]. Finally, in Subsection 3.6 we prove that

$$
b^{*}(1 / 4) \in\left(\frac{951225059}{2609347034}, \frac{258052528}{707875165}\right) \simeq(0.364545247,0.3645452486) .
$$

We use this information to improve the results of [9]. This subsection also serves to show how to use our approach when the problem that we face depends only on one parameter.

At the best of our knowledge, it is the first time that this type of results are obtained for global bifurcation problems. 


\section{The First FAMILY OF QUADRATIC SYSTEMS}

Our starting point is the following theorem:

Theorem 2.1. Consider the system (1),

$$
\left\{\begin{array}{l}
\dot{x}=P(x, y)=y \\
\dot{y}=Q(x, y)=-x+b y+x y-n y^{2},
\end{array}\right.
$$

with $(n, b) \in \mathbb{R}^{2}$. Then there exits a function $b=b^{*}(n)$ such that $b^{*}(-n)=-b^{*}(n)$, $n b^{*}(n)>0$ for $n \neq 0$ for which:

(i) The system has exactly one hyperbolic limit cycle if either $n>0$ and $0<b<$ $b^{*}(n)$ or $n<0$ and $b^{*}(n)<b<0$.

(ii) Otherwise the system has no limit cycles.

See Figure 1 for a numeric plot of the function $b=b^{*}(n)$.

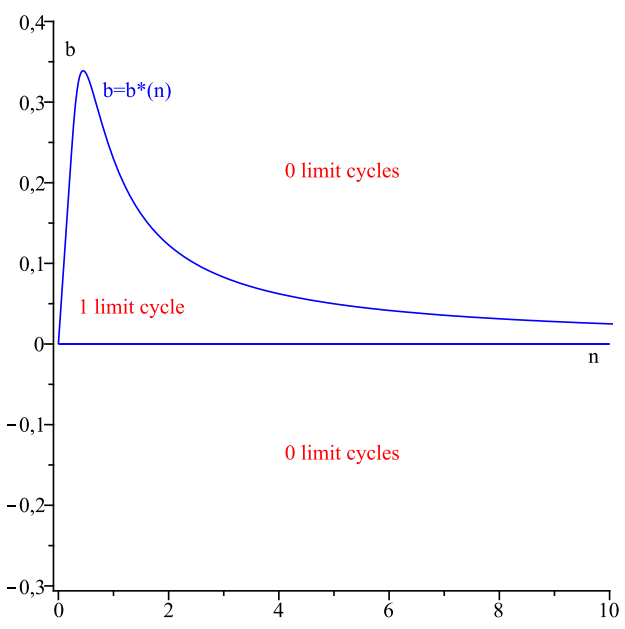

Figure 1. Limit cycles of system (1) for $n>0$. The curve $b=b^{*}(n)$ is obtained numerically.

Essentially its proof follows from [15] and the theory of rotated vector fields $[6,12]$. See also [4] and [15]. The property $b^{*}(-n)=-b^{*}(n)$ is a consequence of the fact that the transformation $(x, y, t) \rightarrow(-x, y,-t)$ changes the sign of $b$ and $n$ in system (1).

The phase portraits of system (1) in the Poincaré sphere appear in [4]. Indeed the phase portrait corresponding to $b=b^{*}(n)$, for $n \neq 0$, corresponds to the one having a heteroclinic connection of the two infinite semi-hyperbolic critical points which are on the directions $y=0$ and $x-n y=0$, see Figure 2. From the above considerations we already know that we can restrict our attention to the region $n>0$ and $b>0$. The origin is the only finite critical point of the system. Moreover the line $y-1=0$ is transversal to the flow associated to this system. A key result for knowing, once $n$ is fixed, if some value $b$ is smaller or bigger than $b^{*}(n)$ is the following lemma. 


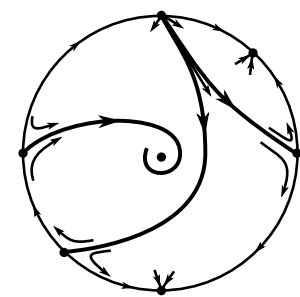

$b \leq 0$

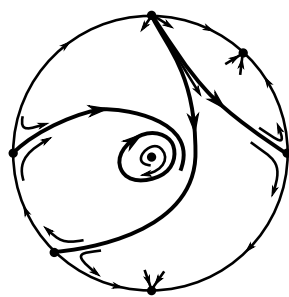

$0<b<b^{*}(n)$



$b=b^{*}(n)$

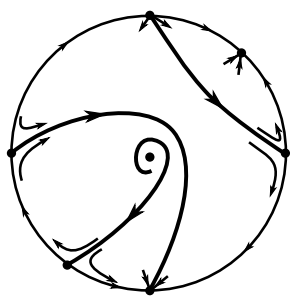

$b^{*}(n)<b<n$

Figure 2. Phase portraits of system (1) for $n>0$ and $b<n$.

Lemma 2.2. Let $\phi(x, y)=q(y) x-p(y)=0$, with $p(y)$ and $q(y)$ polynomials, be an algebraic curve having a non-singular branch connecting the two critical points at infinity of system (1), which correspond with the directions $y=0$ and $x-n y=0$. Assume also that $\phi(0,0)>0$. If for some $n>0$ and $b>0$ it holds that

$$
M(x, y):=\frac{\partial \phi(x, y)}{\partial x} P(x, y)+\left.\frac{\partial \phi(x, y)}{\partial y} Q(x, y)\right|_{\{\phi(x, y)=0\}} \geq 0 \quad(\text { resp. } \leq 0)
$$

and the zeroes of $M$ are isolated, then $b<b^{*}(n)$ (resp. $b>b^{*}(n)$ ), see Figure 3.

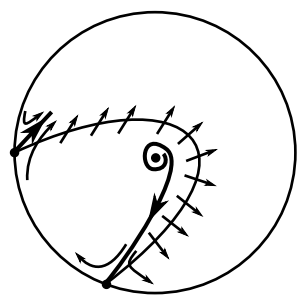

$M<0$



$M>0$

Figure 3. Flow on the curve $\phi(x, y)=0$.

Proof. Consider for instance the case where $M$ is positive or zero. The inequality $M(x, y) \geq 0$ implies that the connected component of the curve $\{\phi(x, y)=0\}$ described in the statement is crossed by the flow of the vector field $(P, Q)$ from the region $\{\phi(x, y)<0\}$ to the region $\{\phi(x, y)>0\}$. Since for $b>0$ the origin is a repellor, by applying the Poincaré-Bendixson Theorem on the sphere to the positive invariant region formed by the curve and a piece of the equator (which is invariant by the flow), we can prove the existence of at least one periodic orbit of system (1). By using Theorem 2.1 we get that $b<b^{*}(n)$, as we wanted to prove. The other case follows similarly.

The main goal of this section is to get good lower and upper bounds for the function $b=b^{*}(n)$.

2.1. A first approach to the bifurcation curve. Our first result takes advantage of a direct application of Lemma 2.2 by taking the curves $\phi(x, y)=0$ as hyperbolas. As can be seen in Figure 4, the curve $b=b^{*}(n)$ is quite well delimited by this simple approach. 




Figure 4 . Observe how the bifurcation curve $b=b^{*}(n)$ of system (1), obtained numerically, lies between the two dashed curves given in Proposition 2.3.

Proposition 2.3. Let $b=b^{*}(n)$ be the bifurcation curve for the quadratic system (1), with $n>0$ and $b>0$. Then, for all $n>0$ :



Proof. Consider $\phi(x, y)=a_{0}+a_{1} x+a_{2} y+x y-n y^{2}$. Clearly, inside this family of hyperbolas there are many of them having the geometrical restrictions described in Lemma 2.2. By computing $M(x, y)$, since on $\phi(x, y)=0$, we can isolate $x$ in terms of $y$, we get that $M$ depends only on $y$. Moreover its numerator is a polynomial of degree 4 in $y$ and its denominator is a polynomial of degree 2 which is a perfect square. There are several ways of forcing it to have a given sign in the region where the connected component of $\phi(x, y)=0$ of our interest lies. For instance, by taking $n>1 / 2$,

$$
a_{0}=\frac{1+4 n^{2}}{8 n^{3}}, a_{1}=-1, a_{2}=-\frac{1+8 n^{2}}{16 n^{3}} \quad \text { and } \quad b=\frac{8 n^{2}-1}{16 n^{3}}
$$

we force this polynomial to be $(y-1)$ times a polynomial of degree two which is a perfect square. Concretely we get that

$$
M(x(y), y)=\frac{\left(4 n^{2}+1\right)\left(4 n^{2} y-1\right)^{2}}{128 n^{6}(y-1)}<0
$$

in the region $\{y<1\}$ and that the hyperbola connects the two infinite critical points, as shown in Figure 3. By using Lemma 2.2 we get that for $n>1 / 2$, $\left(8 n^{2}-1\right) /\left(16 n^{3}\right)>b^{*}(n)$, as we wanted to prove. 
By imposing that the polynomial in the numerator of $M(x(y), y)$ be the product of $y^{2}$ and another polynomial of degree two, which is also a perfect square, we get

$$
\begin{array}{rrr}
a_{0}=\frac{\left(2 n^{2}+1\right)^{2}\left(8 n^{2}+1\right)^{2}}{2 n\left(4 n^{2}+1\right)^{4}}, & a_{1}=-\frac{\left(2 n^{2}+1\right)\left(8 n^{2}+1\right)}{\left(4 n^{2}+1\right)^{2}}, \\
a_{2}=-\frac{\left(2 n^{2}+1\right)\left(8 n^{2}+1\right)}{2 n\left(4 n^{2}+1\right)^{2}}, & b=\frac{n}{4 n^{2}+1} .
\end{array}
$$

Moreover $M(x(y), y)=y^{2} R_{1}^{2}(y) / S_{1}^{2}(y)>0$, where $R_{1}$ and $S_{1}$ are polynomials of degree one in $y$, and $S_{1}(y) \neq 0$ in the region $\{y<1\}$. Again by Lemma 2.2 we get that for all $n>0, n /\left(4 n^{2}+1\right)<b^{*}(n)$.

To end the proof it only remains to show that for $0<n<1 / 2, b^{*}(n)<n$. Indeed this is true for all $n>0$. Notice that the straight line $\{y=1\}$ can not be cut by the periodic orbits of the system. Since, when $b-n \geq 0$,

$$
\operatorname{div}\left(\frac{y}{1-y}, \frac{-x+b y+x y-n y^{2}}{1-y}\right)=\frac{b-2 n y+n y^{2}}{(1-y)^{2}} \geq 0,
$$

by applying the Bendixson-Dulac criterion we get that the system has no limit cycles. Thus $b^{*}(n)<n$, as we wanted to prove.

Remark 2.4. By imposing that the polynomial of degree four appearing in the above proof be a multiple of $y^{4}$ we get another quite satisfactory lower bound for $b=$ $b^{*}(n)$ given by the function $b=\left(4 n^{2}-1\right) /\left(16 n^{3}\right)$. Notice that $\left(4 n^{2}-1\right) /\left(16 n^{3}\right)<$ $n /\left(4 n^{2}+1\right)$.

2.2. Proof of Theorem 1.1. For simplicity, we only give all the details of the proof of the weaker result, which is the one given in item (i), which asserts that for $n>14$,

$$
\left|\frac{1}{4 n}-b^{*}(n)\right|<\frac{1}{8 n^{3}} .
$$

Nevertheless its proof includes all the ingredients for the proof of the other results. To see the main difference for proving the other two statements see the footnote of next page. Before starting the proof we want to comment that although it seems natural to improve item (iii) of the theorem going further in our computations, our results are on the limit of what we can do with the algebraic manipulator that we use (Maple) and the capacity of our computers ${ }^{1}$.

To prove item (i) we will apply in this case the general method described in the previous section. Recall that when $b=b^{*}(n)$ there is a heteroclinic connection between two critical points at infinity of the Poincaré compactification of system (1), see Figure 2. Recall also that the directions corresponding to these critical points are given by the lines $R(x, y):=x Q_{2}(x, y)-y P_{2}(x, y)=0$, where $P_{2}$ and $Q_{2}$ are the quadratic homogeneous parts of $P$ and $Q$, respectively. In our case $R(x, y)=x y(x-n y)$. The behaviour near infinity of the separatrices of these critical points can be easily obtained. For instance the one corresponding to the direction $x-n y=0$, from the center manifold theorem, can be written as

$$
x=\Psi(y)=n y+\psi\left(\frac{1}{y}\right),
$$

\footnotetext{
${ }^{1}$ We have used a computer with $32 \mathrm{~Gb}$ of RAM and $2.4 \mathrm{GHz}$.
} 
with $\psi$ being an analytic function at zero of the form

$$
\psi(w)=\frac{1+n^{2}-n b}{n}\left(1+w+\frac{n^{2}+1}{n^{2}} w^{2}+\frac{\left(n^{2}+2\right)\left(n^{2}+1\right)}{n^{4}} w^{3}+O\left(w^{4}\right)\right) .
$$

Indeed we will use this expansion at much higher order.

Now we search an algebraic curve of the form ${ }^{2}$

$$
x=f(y):=\frac{p_{u}(y)}{q_{v}(y)}=\frac{u_{0}+u_{1} y+u_{2} y^{2}+u_{3} y^{3}+u_{4} y^{4}+n y^{5}}{(y-1)\left(v_{0}+v_{1} y+v_{2} y^{2}+y^{3}\right)}=n y+\sum_{k=0}^{\infty} f_{k} y^{-k},
$$

which is as close as possible to the searched separatrix. This is done by imposing that $\Psi(y)$ and $f(y)$ coincide at the highest order possible at infinity. Concretely, the eight free constants in $f(y)$ are used to vanish the coefficients of $y^{-k}, k=$ $0,1, \ldots, 7$ of $\Psi(y)-f(y)$, obtaining the asymptotic expansion

$$
\Psi(y)-f(y)=\frac{C_{8}(n, b)}{y^{8}}+\frac{C_{9}(n, b)}{y^{9}}+\cdots,
$$

where

$$
C_{8}(n, b)=\frac{\left(-1-n^{2}+n b\right) N_{8}(n, b)}{D_{8}(n, b)},
$$

being

$$
\begin{aligned}
N_{8}(n, b)= & 4 n^{19} b-23 n^{18} b^{2}+70 n^{17} b^{3}-87 n^{16} b^{4}+36 n^{15} b^{5}-n^{18}-231 n^{17} b \\
& +475 n^{16} b^{2}-143 n^{15} b^{3}-100 n^{14} b^{4}+59 n^{16}-2488 n^{15} b+3668 n^{14} b^{2} \\
& -886 n^{13} b^{3}-232 n^{12} b^{4}+567 n^{14}-11151 n^{13} b+10886 n^{12} b^{2}-1284 n^{11} b^{3} \\
& +2561 n^{12}-28350 n^{11} b+16332 n^{10} b^{2}-272 n^{9} b^{3}+7718 n^{10}-44884 n^{9} b \\
& +12264 n^{8} b^{2}+17160 n^{8}-44920 n^{7} b+3648 n^{6} b^{2}+27000 n^{6}-26112 n^{5} b \\
& +27024 n^{4}-6624 n^{3} b+14976 n^{2}+3456
\end{aligned}
$$

and

$$
\begin{aligned}
D_{8}(n, b)= & -9 n^{9} b+9 n^{8} b^{2}-33 n^{7} b+8 n^{6} b^{2}+2 n^{8}+16 n^{6} \\
& -52 n^{5} b+50 n^{4}-28 n^{3} b+60 n^{2}+24 .
\end{aligned}
$$

Therefore, given $n=\bar{n}$, some of the values of $b$ satisfying $N_{8}(\bar{n}, b)=0$ could be a good candidate to approximate $b^{*}(\bar{n})$. It is not difficult to prove that one of the connected components of $N_{8}(n, b)=0$ has an asymptotic expansion at infinity of the form $b=\frac{1}{4 n}+\frac{3}{64 n^{3}}+\cdots$. From this expansion, and taking into account the results of Proposition 2.3, we decide to fix

$$
b=\frac{1}{4 n}+\frac{\alpha}{n^{3}},
$$

\footnotetext{
${ }^{2}$ To prove statements (ii) and (iii), the only difference is that we take a rational function with the same structure but having higher degrees in both the numerator and the denominator. Concretely, having respectively degrees 6 and 5 for (ii), and 8 and 7 for (iii).
} 
for some $\alpha$ to be determined afterwards and the $u_{i}=u_{i}(n, \alpha)$ and $v_{i}=v_{i}(n, \alpha)$ obtained above. Taking $\phi_{n, \alpha}(x, y)=q_{v}(y) x-p_{u}(y)$ in Lemma 2.2 we get that

$$
M_{n, \alpha}(f(y), y)=t(n, \alpha) \frac{\sum_{i=0}^{4} r_{i}(n, \alpha) z^{i}}{z\left(\sum_{i=0}^{3} s_{i}(n, \alpha) z^{i}\right)^{2}}, \quad \text { where } \quad z=1-y,
$$

and $f(y)=p_{u}(y) / q_{v}(y)$. In the above expression all the functions $r_{i}(n, \alpha)$ are polynomials in $n$ of degree 40 , and all $s_{i}(n, \alpha)$ are also polynomials in $n$. The function $t(n, \alpha)$ is a rational function, which is always negative, and the coefficients of $n^{40}$ for each $r_{i}(n, \alpha)$, for $i=0, \ldots, 4$ are, respectively,

$$
-\frac{5}{256}-\frac{\alpha}{4},-\frac{3}{64}-\alpha,-\frac{3}{128}-\frac{3 \alpha}{2}, \frac{1}{64}-\alpha, \frac{3}{256}-\frac{\alpha}{4} .
$$

When $\alpha=1 / 8$ (resp. $-1 / 8$ ) all the expressions of the above list are negative (resp. positive). Hence we can assure that for this value of $\alpha$ there exist $n_{0}^{ \pm}$ such that for all $z>0$ and all $n>n_{0}^{+}$(resp. $n>n_{0}^{-}$) the numerator of (3) is positive (resp. negative). Similarly it can be proved that the denominator never vanishes on the same region. Indeed it can be seen that the biggest value of $n$ which vanishes some of the eighteen functions, $r_{i}(n, \pm 1 / 8), i=0, \ldots, 4$, and $s_{i}(n, \pm 1 / 8), i=0, \ldots, 3$ corresponds to a zero of a factor of $r_{4}(n, 1 / 8)$. This factor is

$$
\begin{aligned}
& 2560 n^{16}-446720 n^{14}-2294624 n^{12}+1116256 n^{10}+33662656 n^{8} \\
& +98872176 n^{6}+131940378 n^{4}+85765842 n^{2}+21990713,
\end{aligned}
$$

and its biggest zero is approximately 13.397. In short, by using for instance the Sturm algorithm, it can be proved that for $\alpha=1 / 8$ (resp. $\alpha=-1 / 8), n>13.4$ and $z>0$ it holds that the expression in (3) is positive (resp. negative). By using Lemma 2.2 the theorem follows.

Remark 2.5. It is clear that our method to choose the curve $\phi(x, y)=0$, which is one of the key points of our approach, that consists in imposing that it coincides as much as possible with one of the separatrices, can be done by using the separatrix of the other infinite critical point. Also it can be imposed that the curve $\phi(x, y)=0$ coincides in both extremes, i.e. simultaneously as much as possible, with both separatrices. Although we obtain also other bounds for $b=b^{*}(n)$, the ones given in Theorem 1.1 are, at least for $n$ big, better than the ones obtained by using these slightly different approaches.

\section{The Bogdanov-Takens system}

For the Bogdanov-Takens system all its bifurcation diagram, including the number of limit cycles is known, see for instance $[3,8,10,13]$. We summarize these results about limit cycles in the next theorem:

Theorem 3.1. Consider the system (2),

$$
\left\{\begin{array}{l}
\dot{x}=y \\
\dot{y}=-n+b y+x^{2}+x y
\end{array}\right.
$$


with $n$ and $b$ real numbers. Then it has a limit cycle (which is unique and hyperbolic) if and only if

$$
n>0 \text { and } b^{*}(n)<b<\sqrt{n} .
$$

Here the function $b^{*}: \mathbb{R}^{+} \rightarrow \mathbb{R}$ gives the curve $b=b^{*}(n), n \geq 0$, where the phase portrait of (2) has a homoclinic loop, which is hyperbolic and unstable. Moreover

$$
b^{*}(n)=\frac{5}{7} \sqrt{n}+O(n), \quad n \sim 0 .
$$

See Figure 5 for a numeric plot of $b=b^{*}(n)$.

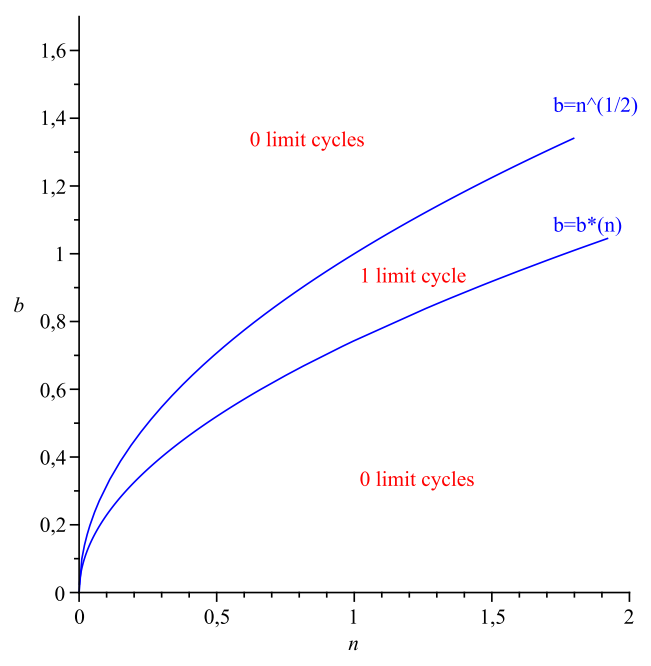

Figure 5. Limit cycles of the Bogdanov-Takens system. The curve $b=b^{*}(n)$ is obtained numerically.

Similarly that in the previous section our main goal is to give analytic information on the function $b=b^{*}(n)$ above. Following [3, 8] we know that, fixed $n>0$, a hyperbolic unstable limit cycle is created when $b=\sqrt{n}$, via a Hopf bifurcation, that this limit cycle increases size when $b$ decreases and disappears for some value $b=b^{*}(n)$, for which the system presents a homoclinic unstable saddle loop. This behavior of the limit cycle is due to the fact that the system is a semi-complete family of rotated vector fields with parameter $b$, see for instance [6, 12], see Figure 6 . The uniqueness and hyperbolicity of the limit cycle is proved for instance in [10].

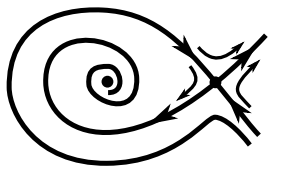

$b<b^{*}(n)$



$b=b^{*}(n)$

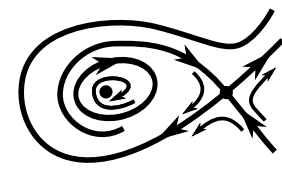

$b^{*}(n)<b<\sqrt{n}$



$b \geq \sqrt{n}$

Figure 6. Saddle loop and Hopf bifurcations for the BogdanovTakens system. 
3.1. A first approach to $b^{*}(n)$. When $n \geq 0$, instead of working with the expression (2), by using the translation $x_{1}=x-\sqrt{n}, y_{1}=y$, after dropping the subindexes, we obtain the equivalent system

$$
\left\{\begin{array}{l}
\dot{x}=y \\
\dot{y}=2 \sqrt{n} x+(b+\sqrt{n}) y+x^{2}+x y
\end{array}\right.
$$

A simple first estimation of the function $b=b^{*}(n)$ is given in next lemma:

Lemma 3.2. Let $b=b^{*}(n)$ be the value corresponding to a homoclinic saddle loop for system (2), then

$$
\max (-\sqrt{n}, \sqrt{n}-1)<b^{*}(n)<\sqrt{n} .
$$

Proof. We work with the expression (4) of the Bogdanov-Takens system. Recall that the limit cycle is born, via a Hopf bifurcation, when $b=\sqrt{n}$ and $b$ decreases. Hence, by using the non-intersection property of the limit cycles of a semi-complete family of rotated vector fields it suffices to prove that, when either $b=\sqrt{n}-1$ or $b=-\sqrt{n}$, system (4) has no limit cycles. When $b=\sqrt{n}-1$, note that the line $x+y=0$ is invariant by the flow of (4), because

$$
\frac{\partial L(x, y)}{\partial x} y+\frac{\partial L(x, y)}{\partial y}\left(2 \sqrt{n} x+(b+\sqrt{n}) y+x^{2}+x y\right)=(2 \sqrt{n}+x) L(x, y),
$$

where $L(x, y)=x+y$. In other words, $L(x, y)$ is an invariant algebraic curve with cofactor $K(x, y):=2 \sqrt{n}+x$.

Assume that, when $b=\sqrt{n}-1$, system (4) has a limit cycle $\Gamma=\{(x(t), y(t))\}$, with period $T$. Since the divergence of the system is $b+\sqrt{n}+x=2 \sqrt{n}-1+x$, the characteristic exponent of $\Gamma$ is

$$
\kappa:=\int_{0}^{T}(2 \sqrt{n}-1+x(t)) d t
$$

On the other hand notice that from (5) we get that

$$
\int_{0}^{T}(2 \sqrt{n}+x(t)) d t=\int_{0}^{T} \frac{\frac{d}{d t} L(x(t), y(t))}{L(x(t), y(t))}=0 .
$$

Hence $\kappa=-T$ and so $\Gamma$ would be a hyperbolic stable limit cycle. This fact is in contradiction with the known results, see for instance Figure 6. Hence the limit cycle does not exist in this case.

To end the proof let us show that $b^{*}(n)>-\sqrt{n}$. It is well-known that the stability of a hyperbolic saddle loop is given by the sign of the divergence at the saddle point, see $[1$, Chap. XI]. In our case, the divergence at the saddle point is $b^{*}(n)+\sqrt{n}$. For the Bogdanov Takens system (2), when the loop exists it is hyperbolic and unstable. Hence it holds that $b^{*}(n)+\sqrt{n}>0$, as we wanted to prove.

3.2. Proof of Theorem 1.2.(i). We introduce the new parameter $m=n^{1 / 4}$ and we consider the equivalent system given in (4),

$$
\left\{\begin{array}{l}
\dot{x}=y \\
\dot{y}=2 m^{2} x+\left(b+m^{2}\right) y+x^{2}+x y
\end{array}\right.
$$


We will prove our result in a constructive way. Indeed our first steps could be skipped but we believe that they are useful to understand the way we have obtained the approximations for $b^{*}(n)$.

System (6) has a focus or a node at the point $\left(-2 m^{2}, 0\right)$ and a saddle point at the origin. The linear approximation to its separatrices at the origin is given by the two lines:

$$
C_{2}(x, y):=-2 m^{2} x^{2}-\left(b+m^{2}\right) x y+y^{2}=0,
$$

or equivalently, $y=a_{1} x$, where $a_{1}$ is one of the values

$$
a_{1}^{ \pm}:=\frac{b+m^{2} \pm \sqrt{\left(b+m^{2}\right)^{2}+8 m^{2}}}{2} .
$$

As in the proof of Theorem 1.1 we start by finding the first terms in the Taylor expansion of the separatrices of a critical point. In this occasion the critical point is the origin. In its neighborhood we can write these separatrices as

$$
y=\Psi(x):=\sum_{k=1}^{\infty} a_{k} x^{k},
$$

where $a_{1}$ is one of the values $a_{1}^{ \pm}$, or in other words, $C_{2}\left(1, a_{1}\right)=0$. The next terms can be found recurrently by imposing that

$$
\frac{\partial(y-\Psi(x))}{\partial x} y+\left.\frac{\partial(y-\Psi(x))}{\partial y}\left(2 m^{2} x+\left(b+m^{2}\right) y+x^{2}+x y\right)\right|_{y=\Psi(x)} \equiv 0 .
$$

We have, for instance,

$$
\begin{aligned}
a_{2} & =\frac{-1-a_{1}}{b-3 a_{1}+m^{2}}, & a_{3} & =\frac{a_{2}\left(2 a_{2}-1\right)}{b-4 a_{1}+m^{2}}, \\
a_{4} & =\frac{a_{3}\left(-1+5 a_{2}\right)}{b-5 a_{1}+m^{2}}, & a_{5} & =\frac{3 a_{3}^{2}-a_{4}+6 a_{2} a_{4}}{b-6 a_{1}+m^{2}} .
\end{aligned}
$$

We remark that $b-k a_{1}+m^{2}$ never vanishes for $k>1$.

Now we consider a cubic algebraic curve of the form

$$
C(x, y):=C_{2}(x, y)+c_{3,0} x^{3}+c_{2,1} x^{2} y+c_{1,2} x y^{2}+c_{0,3} y^{3}=0
$$

and we search the four free coefficients by imposing that $\{C=0\}$ be as close as possible to the separatrices of the saddle point. In particular notice that the quadratic terms of $C$ imply that this curve is tangent to both separatrices. This can be done by imposing that the function

$$
F(x):=C(x, \Psi(x))=\sum_{k=3}^{\infty} f_{k}(m, b) x^{k},
$$

be as flat as possible at the origin. By choosing suitable $c_{i, j}$ in terms of $b$ and $m$ we get that

$$
F(x)=f_{7}(m, b) x^{7}+O\left(x^{8}\right) .
$$

After some cumbersome computations, done with an algebraic manipulator, we obtain that all the solutions of the equation $f_{7}(m, b)=0$ are contained in the algebraic curve 


$$
\begin{aligned}
& (2+b)\left(b+1-m^{2}\right)\left(b^{2}+9 m^{2}+2 b m^{2}+m^{4}\right)^{2} \times \\
& \left(3726 m^{20}+21951 m^{18}+25110 b m^{18}-671220 m^{16}+68040 b^{2} m^{16}\right. \\
& +109512 b m^{16}+169452 b^{2} m^{14}+87480 b^{3} m^{14}-3005247 m^{14} \\
& -1882701 b m^{14}-1560330 b^{2} m^{12}-40176 b^{3} m^{12}-5046048 b m^{12} \\
& +17286120 m^{12}+34020 b^{4} m^{12}-703539 b^{3} m^{10}-432702 b^{4} m^{10} \\
& -2360050 m^{10}-1021503 b^{2} m^{10}-39074153 b m^{10}-47628 b^{5} m^{10} \\
& -848112 b^{3} m^{8}+31843300 b^{2} m^{8}+640000 m^{8}-530064 b^{5} m^{8} \\
& -1329192 b^{4} m^{8}-68040 b^{6} m^{8}+9121240 b m^{8}-1181763 b^{5} m^{6} \\
& -29160 b^{7} m^{6}-1792000 b m^{6}-238788 b^{6} m^{6}-2892813 b^{4} m^{6} \\
& -11432588 b^{2} m^{6}-18903846 b^{3} m^{6}-89544 b^{5} m^{4}+1254400 b^{2} m^{4} \\
& +22032 b^{7} m^{4}+56862 b^{6} m^{4}+4247140 b^{4} m^{4}+2430 b^{8} m^{4} \\
& +4429720 b^{3} m^{4}+974859 b^{6} m^{2}+5670 b^{9} m^{2}+243950 b^{4} m^{2} \\
& +54999 b^{8} m^{2}+307107 b^{7} m^{2}+863815 b^{5} m^{2}+1296 b^{10} \\
& \left.+13608 b^{9}+42984 b^{8}+39000 b^{7}+11400 b^{6}\right)=0 .
\end{aligned}
$$

The branches passing through the origin of the algebraic curve given above are

$$
b=\frac{5}{7} m^{2}+\frac{72}{2401} m^{4} \pm \frac{4428}{2401} \sqrt{2} m^{5}+O\left(m^{6}\right) .
$$

Hence to continue our study we decide to fix

$$
b=\frac{5}{7} m^{2}+\frac{72}{2401} m^{4}+\alpha m^{5}
$$

with $\alpha$ to be given afterwards.

With $b$ given in (9) we will try to fix $\alpha$ in such a way that the curve $C$ be without contact in the negative half-plane $\{(x, y): x<0\}$ where the saddle separatrices lie. To impose this constrain we compute the resultant of $C(x, y)$ and

$$
D(x, y):=\frac{\partial C(x, y)}{\partial x} y+\frac{\partial C(x, y)}{\partial y}\left(2 m^{2} x+\left(b+m^{2}\right) y+x^{2}+x y\right),
$$

with respect to $y$. This a very huge task. After many computations ${ }^{3}$, done again with an algebraic manipulator, we obtain that

$$
P(x ; \alpha, m):=\operatorname{Res}(C, D, y)=x^{10} m^{36}\left(r_{0}(\alpha, m)+r_{1}(\alpha, m) x+r_{2}(\alpha, m) x^{2}\right),
$$

where for $i=0,1,2$, it holds that

$$
r_{i}(\alpha, m)=r_{i}^{0}(\alpha, m)+r_{i}^{1}(\alpha, m) \Delta(\alpha, m),
$$

\footnotetext{
${ }^{3}$ Notice that this is a main difference between the proof of this theorem and the one of Theorem 1.1, because there the algebraic curve considered is of the form $x=f(y)$, and so the problem goes easily to a one variable problem, while in this case we have to evaluate the resultant of two polynomials.
} 
where

$$
\begin{aligned}
\Delta(\alpha, m):= & \left(5764801 \alpha^{2} m^{8}+345744 \alpha m^{7}+5184 m^{6}+19765032 \alpha m^{5}\right. \\
& \left.+592704 m^{4}+16941456 m^{2}+46118408\right)^{1 / 2}
\end{aligned}
$$

and all the functions $r_{i}^{j}(\alpha, m)$ are polynomials in $\alpha$ and $m$. Solving the quadratic equation

$$
p_{2}(x ; \alpha, m):=r_{0}(\alpha, m)+r_{1}(\alpha, m) x+r_{2}(\alpha, m) x^{2}=0,
$$

we obtain the solutions

$$
\begin{aligned}
x=x^{+}(\alpha, m) & =\frac{-r_{1}(\alpha, m)+\sqrt{r_{1}^{2}(\alpha, m)-4 r_{0}(\alpha, m) r_{2}(\alpha, m)}}{2 r_{2}(\alpha, m)} \\
& =\left(-\frac{63}{25}+\frac{16807}{18450} \sqrt{2} \alpha+\frac{282475249}{326786400} \alpha^{2}\right) m^{2}+O\left(m^{3}\right), \\
x=x^{-}(\alpha, m) & =\frac{-r_{1}(\alpha, m)-\sqrt{r_{1}^{2}(\alpha, m)-4 r_{0}(\alpha, m) r_{2}(\alpha, m)}}{2 r_{2}(\alpha, m)} \\
& =\frac{247107}{304580}+O(m) .
\end{aligned}
$$

Notice that the two roots of the coefficient of $m^{2}$ in $x^{+}(\alpha, m)$ are

$$
\alpha=\alpha^{+}:=\frac{13284}{16807} \sqrt{2} \simeq 1.12, \quad \alpha=\alpha^{-}:=-\frac{4428}{2401} \sqrt{2} \simeq-2.61 .
$$

Hence, taking for instance $\alpha= \pm 11 / 4= \pm 2.75$ we obtain two values of $\alpha$ for which, for $m$ small enough, the two roots of the quadratic polynomial in $p_{2}(x ; \alpha, m)$ are positive and so the curve $C$ is without contact, for $m$ small enough, in $\{(x, y)$ : $x<0\}$. To know until which values of $m$ this last property holds we fix $\alpha= \pm 11 / 4$ and study the signs of the functions $r_{i}( \pm 11 / 4, m), i=0,1,2$.

As an example we give some details for

$$
r_{2}(-11 / 4, m)=r_{2}^{0}(-11 / 4, m)+r_{2}^{1}(-11 / 4, m) \Delta(-11 / 4, m) .
$$

It can be seen that the degrees of $r_{2}^{0}(-11 / 4, m)$ and $r_{2}^{1}(-11 / 4, m)$ are 180 and 184 , respectively. Notice that the above expression does not change sign when

$$
\left(r_{2}^{0}(-11 / 4, m)\right)^{2}-\left(r_{2}^{1}(-11 / 4, m)\right)^{2}(\Delta(-11 / 4, m))^{2}
$$

does not change sign. Hence we have to study the zeroes of this function which is a polynomial in $m$. This can be done analytically by using its Sturm sequence. The smallest positive root of the polynomial (11) which also vanishes (10) is $m \simeq 0.40289$. Hence we have proved that for $m \in(0,4 / 10)$, it holds that $r_{2}(-11 / 4, m)<0$. Similarly we can prove that, on the interval $(0,4 / 10)$,

$$
\begin{array}{lll}
r_{0}(11 / 4, m)<0, & r_{1}(11 / 4, m)>0, & r_{2}(11 / 4, m)<0, \\
r_{0}(-11 / 4, m)<0, & r_{1}(-11 / 4, m)>0, & r_{2}(-11 / 4, m)<0 .
\end{array}
$$

As a consequence, the curve $C$ with $b$ given in (9), $\alpha= \pm 11 / 4$, and $m \in(0,4 / 10)$ is without contact in $\{(x, y): x<0\}$. It is easy to prove that for $m$ sufficiently small the curve $C$ has a loop in the half-plane $\{(x, y): x<0\}$. Moreover with the same type of algebraic methods that we have used above it can be seen that the loop also exists in the interval $m \in(0,4 / 10)$. Furthermore, in this half-plane 
the loop is crossed transversally by the flow of (6), inwards when $\alpha=-11 / 4$ and outwards when $\alpha=11 / 4$. Hence we have proved that when

$$
b=b^{ \pm}(m):=\frac{5}{7} m^{2}+\frac{72}{2401} m^{4} \pm \frac{11}{4} m^{5},
$$

and $m \in(0,4 / 10)$ it holds that $b^{-}(m)<b^{*}(m)<b^{+}(m)$. From these inequalities the statement (i) of the theorem follows by noticing that $(4 / 10)^{4}=16 / 625>1 / 40$.

3.3. Proof of Theorem 1.2.(ii). Clearly the ideas of the method used to prove Theorem 1.2.(i) can be applied when the curve $C$ given in (8) is taken of higher degree. Consider now the algebraic curve

$$
C_{4}(x, y):=C_{2}(x, y)+\sum_{3 \leq i+j \leq 4} c_{i, j} x^{i} y^{j}=0 .
$$

By employing the same procedure applied to the cubic algebraic curve we obtain the following relation between $b$ and $n=m^{4}$ :

$$
b=\frac{5}{7} n^{1 / 2}+\frac{72}{2401} n-\frac{30024}{45294865} n^{3 / 2}-\frac{2352961656}{11108339166925} n^{2}+O\left(n^{5 / 2}\right) .
$$

By using the variables $n$ and $b$, the necessary calculations to obtain analogous results, for a given interval $\left[0, n_{0}\right]$, to those obtained in Theorem 1.2.(i) for the cubic curve are beyond our computational capacity. In particular we can not obtain a compact expression of the resultant that would give a proof that $C_{4}$ is without contact in the half-plane $\{(x, y): x<0\}$. Nevertheless, once $n$ is fixed we can perform all the computations and prove for instance that for $n=1 / 20$,

$$
\left|\frac{5}{7} n^{1 / 2}+\frac{72}{2401} n-\frac{30024}{45294865} n^{3 / 2}-b^{*}(n)\right|<10^{-4} n^{7 / 4},
$$

and for $n=1 / 100$,

$$
\left|\frac{5}{7} n^{1 / 2}+\frac{72}{2401} n-\frac{30024}{45294865} n^{3 / 2}-\frac{2352961656}{11108339166925} n^{2}-b^{*}(n)\right|<10^{-5} n^{9 / 4} .
$$

In any case, we will prove that the expression (13) gives us, at least locally, the function $b^{*}(n)$. This can be done by introducing the new variables $B>0$ and $M>0$ as

$$
B=\frac{b+m^{2}}{2}, \quad M^{2}=\frac{\left(b+m^{2}\right)^{2}+8 m^{2}}{4} .
$$

This change of variables is motivated by equation (7). Notice that by using them, $a_{1}^{ \pm}=B \pm M$. Now we repeat all the procedure developed in the previous subsection but with the curve $C_{4}(x, y)=0$ given in (12) and these new variables. By forcing the curve $C_{4}(x, y)=0$ to coincide as much as possible with both separatrices of the saddle point (four conditions of contact with one of them and five with the other one) we determine all the parameters of the quartic. After that we obtain the relation

$$
B=\frac{3}{7} M^{2}-\frac{180}{2401} M^{4}+\frac{2366307}{90589730} M^{6}-\frac{505643614857}{44433356667700} M^{8}+O\left(M^{9}\right),
$$

that ensures one more level of closeness between one of the separatrices and the algebraic curve, for $M$ sufficiently small. 
Fortunately, with these new variables it is now possible to explicitly get the resultant $P(x ; M, B)$, between $C_{4}(x, y)$ and

$$
D_{4}(x, y):=\frac{\partial C_{4}(x, y)}{\partial x} y+\frac{\partial C_{4}(x, y)}{\partial y}\left(2 m^{2} x+\left(b+m^{2}\right) y+x^{2}+x y\right),
$$

with respect to $y$. We obtain that

$$
P(x ; M, B):=\operatorname{Res}\left(C_{4}, D_{4}, y\right)=x^{14}\left(S_{0}(M, B) \sum_{i=0}^{5} T_{i}(M, B) x^{i}\right),
$$

where $S_{0}$ and $T_{i}, i=0, \ldots, 5$ are rational functions in $B$ and $M$. To have an idea of their complexity we introduce the following notation: we will say that a rational function $S(M, B)$ is of type $\{i, j\} /\{k, l\}$ if after simplifying it, its numerator has monomials of degree between $i$ and $j$ and its denominator between $k$ and $l$. Then

$$
\begin{array}{ll}
S_{0} \text { is }\{0,1\} /\{114,132\}, & \\
T_{0} \text { is }\{91,120\} /\{0,0\}, & T_{1} \text { is }\{109,143\} /\{20,24\}, \\
T_{2} \text { is }\{124,162\} /\{37,44\}, & T_{3} \text { is }\{137,179\} /\{52,62\}, \\
T_{4} \text { is }\{137,179\} /\{53,63\}, & T_{5} \text { is }\{137,180\} /\{55,65\} .
\end{array}
$$

Note that six of the above functions are singular at $B=M=0$. Inspired by (15) we express $B$ in terms of $M$ and $\alpha$ as

$$
B=\beta(M, \alpha):=\frac{3}{7} M^{2}-\frac{180}{2401} M^{4}+\frac{2366307}{90589730} M^{6}-\frac{505643614857}{44433356667700} M^{8}+\alpha M^{9},
$$

where $\alpha$ is an arbitrary parameter to be determined later. We obtain that

$$
\left.\frac{P(x ; \beta(M, \alpha), M)}{x^{14}}\right|_{M=0}=\sum_{i=0}^{5} P_{i}(\alpha) x^{i},
$$

where now all the functions $P_{i}, i=0,1, \ldots, 5$ are polynomials in $\alpha$ with degrees $\{2,5\},\{0,6\},\{0,11\},\{0,15\},\{0,15\}$, and we use a notation similar to above. We remark that to have the cancelations between the respective numerators and denominators in the expression of $P$, that allow us to evaluate it at $M=0$, it is necessary to take $B$ as in the expression (16).

The polynomial $P_{0}$ is

$$
\begin{aligned}
P_{0}(\alpha)=k \alpha^{2}( & 296751659628833594552482011388242366232495503625 \alpha^{3} \\
& +719549554938315584569470362390245200816649200 \alpha^{2} \\
& +532161050006783873283311272385459961077760 \alpha \\
& +112647235678813465306115059636253208576),
\end{aligned}
$$

for some positive integer $k$. It has $\alpha=0$ as a double root and three negative simple roots, which are approximately $-0.001166,-0.000895$ and -0.000364 . In particular

$$
P_{0}\left(-2500^{-1}\right)<0 \text { and } P_{0}\left(2500^{-1}\right)>0 .
$$

If we fix any of the two values $\alpha^{ \pm}= \pm 2500^{-1}$ it holds that $P\left(x ; \beta\left(M, \alpha^{ \pm}\right), M\right)$ does not vanish if $M$ and $|x| \neq 0$ are small enough. This implies that shrinking, if 
necessary, the value of $M$ we obtain that the oval of the algebraic curve $C_{4}(x, y)=$ 0 , which lies in the negative half plane $\{x<0\}$ and starts and ends at the origin, is without contact with the flow of the system (except at $(0,0)$ ). Notice that this oval is born from the cusp point existing when $M=0$ and $B=\beta\left(\alpha^{ \pm}, 0\right)=0$.

The fact that the resultant between $C_{4}$ and $D_{4}$ has different sign when $\alpha=\alpha^{+}$ and when $\alpha=\alpha^{-}$induces to think that the flow crosses the oval outwards in one case of plus sign and inwards in the other one. To prove this fact it suffices to check this property, for each case, on a single point of the oval. We have chosen the cutting point of the oval of $C_{4}(x, y)=0$ and the $x$-axis.

The four points of the curve $C_{4}(x, y)=0$ on the $x$-axis are 0 , which is a double one, and

$$
\begin{aligned}
x_{1}(\alpha, M)= & -\frac{3}{2} M^{2}+\frac{99}{392} M^{4}-\frac{14661}{168070} M^{6}+\frac{1097361567}{28988713600} M^{8} \\
& -\frac{573369660516777}{3110334966739000} M^{10}+\left(\frac{429}{1280} \alpha-\frac{1360220314860156764457}{758276772825613000960000}\right) M^{11}+O\left(M^{12}\right), \\
x_{2}(\alpha, M)= & -\frac{3266440450}{1212150477} \\
& +\left(-\frac{688506579126746016577}{52895116040087791044}+\frac{868315765444642387622750}{357042033270592589547} \alpha\right) M+O\left(M^{2}\right) .
\end{aligned}
$$

Notice that the point on the loop is $\left(x_{1}(\alpha, M), 0\right)$. After many computations we obtain that

$$
\begin{aligned}
D_{4}\left(x_{1}(\alpha, M), 0\right)= & \frac{37182801006000}{184877} \alpha M^{11} \\
& -\left(\frac{1233580648740454800}{3107227739} \alpha+\frac{644635919327316538269855546}{575604427043451909103}\right) M^{12}+O\left(M^{13}\right) .
\end{aligned}
$$

Hence the sign on $C_{4}(x, y)=0$ of $D_{4}$ is different for $\alpha=\alpha^{+}>0$ and for $\alpha=\alpha^{-}<0$, as we wanted to prove. Therefore, for $M$ small enough $^{4}$, the value of $B$ corresponding to the existence of the homoclinic loop, say $B^{*}(M)$, satisfies

$$
\beta\left(M,-2500^{-1}\right)<B^{*}(M)<\beta\left(M, 2500^{-1}\right) .
$$

Collecting all the above results and writing expression (15) in the old variables $b$ and $n$ we get the proof of Theorem 1.2.(ii).

To get an idea of how far the approximation given in Theorem 1.2.(ii) works we compare it with a numerical approximation of the bifurcation curve. Concretely we have obtained a numerical approximation, working with precision $10^{-16}$, of $b=b^{*}(n)$ for $n \in\left(0,10^{-2}\right)$ by using a Taylor's method for solving the differential equation. If we denote it by $b=b_{\text {num }}^{*}(n)$ it holds that

$$
\left|\frac{5}{7} n^{1 / 2}+\frac{72}{2401} n-\frac{30024}{45294865} n^{3 / 2}-\frac{2352961656}{11108339166925} n^{2}-b_{\text {num }}^{*}(n)\right|<6 \times 10^{-10},
$$

for $n \in\left(0,10^{-2}\right)$.

\footnotetext{
${ }^{4}$ To determine an explicit value of $M$ until which the result holds we could try a similar study to the one done in the previous subsection, but it would be extremely long and tedious.
} 
3.4. More terms in $b^{*}(n)$. By applying again the procedure described in the previous subsections, but starting with a sextic algebraic curve

$$
C_{6}(x, y):=C_{2}(x, y)+\sum_{3 \leq i+j \leq 6} c_{i, j} x^{i} y^{j}=0
$$

we arrive to a relation similar to (15) that writes as:

$$
\begin{aligned}
B= & \frac{3}{7} M^{2}-\frac{180}{2401} M^{4}+\frac{2366307}{90589730} M^{6}-\frac{505643614857}{44433356667700} M^{8}+\frac{121044460222851597}{21794117111940173000} M^{10} \\
& -\frac{75409774306549331412249}{25960934362572014675870000} M^{12}+\frac{344552497352535858777709804917}{216470837823465107132247097100000} M^{14} \\
& -\frac{43306460616773431694096161799928995367}{48013140478550999259657196328505023800000} M^{16} \\
& +\frac{91720311301427439675156623493153846098504753619}{174952692015527559148011719369634259959684998000000} M^{18} \\
& -\frac{990748106733217809261982123885784358373281388289053276929}{3187507017704227098311349623537514328886463857480747900000000} M^{20} \\
& +\frac{2174773094339151212022525670857933567647566708078598123403162358137}{11614798115838021630069501971110592530190950393238633306372059000000000} M^{22} \\
& +O\left(M^{24}\right) .
\end{aligned}
$$

From it and the change of variables (14) we obtain the following relation between $b$ and $n$,

$$
\begin{aligned}
b^{*}= & \frac{5}{7} n^{1 / 2}+\frac{72}{2401} n-\frac{30024}{45294865} n^{3 / 2}-\frac{2352961656}{11108339166925} n^{2}+\frac{161066618396136}{2724264638992521625} n^{5 / 2} \\
& -\frac{28575844096870898712}{1622558397660750917241875} n^{3}+\frac{37409973403083644863711656}{6764713681983284597882721784375} n^{7 / 2} \\
& -\frac{1301593321483486009213262204378664}{750205319977359363432143692632890996875} n^{4} \\
& +\frac{7506334550193086288190421267268866218707352}{1366817906371309055843841557575267655935039046875} n^{9 / 2} \\
& -\frac{21889610833333347178341822657596953981848275462851032}{12451199287907137102778709466943415347212749443284171484375} n^{5} \\
& +\frac{1289326941251660725073133052778275691207040442626311930438856}{22685152569996135996229496037325376035529199986794205676507927734375} n^{11 / 2} \\
& +O\left(n^{6}\right) .
\end{aligned}
$$

We believe that it is an improvement of the expression of $b^{*}(n)$ given in Theorem 1.2.(ii), with seven new terms of the expansion of $b^{*}(n)$, but we have not been able to prove this fact due to the complexity of the necessary algebraic calculations. 
Notice that the prime decompositions of the denominators of (17) have a nice and regular structure:

$$
\begin{aligned}
7 & =7, \\
2401 & =7^{4}, \\
45294865 & =5 \cdot 7^{7} \cdot 11, \\
11108339166925 & =5^{2} \cdot 7^{10} \cdot 11^{2} \cdot 13, \\
2724264638992521625 & =5^{3} \cdot 7^{13} \cdot 11^{3} \cdot 13^{2}, \\
1622558397660750917241875 & =5^{4} \cdot 7^{15} \cdot 11^{4} \cdot 13^{3} \cdot 17, \\
67647136819832845978827217843756 & =5^{5} \cdot 7^{18} \cdot 11^{5} \cdot 13^{4} \cdot 17^{2}, \\
750205319977359363 \cdots 92632890996875 & =5^{5} \cdot 7^{22} \cdot 11^{6} \cdot 13^{5} \cdot 17^{3} \cdot 19, \\
136681790637090558 \cdots 55935039046875 & =5^{6} \cdot 7^{25} \cdot 11^{7} \cdot 13^{6} \cdot 17^{4} \cdot 19^{2} \cdot 23, \\
124511992879137102 \cdots 43284171484375 & =5^{8} \cdot 7^{28} \cdot 11^{8} \cdot 13^{7} \cdot 17^{5} \cdot 19^{3} \cdot 23^{2}, \\
226851525699359962 \cdots 76507927734375 & =5^{9} \cdot 7^{31} \cdot 11^{9} \cdot 13^{8} \cdot 17^{6} \cdot 19^{4} \cdot 23^{3},
\end{aligned}
$$

where the small irregularity with the number of fives and sevens could be produced by some cancelations with the respective numerators. Unfortunately no regularity appears in the numerators.

This regularity in the denominators of the asymptotic expansion of $b=b^{*}(n)$ at the origin could give some clues about a possible closed form expression of this function but, by the moment, we have not been able to obtain it.

3.5. Relation between our results and Perko's approach. In [13], the author faces the problem of the global bifurcation diagram, on the Poincaré sphere, of a different representation of the Bogdanov-Takens system. Concretely he considers the system

$$
\left\{\begin{array}{l}
\dot{x}=y, \\
\dot{y}=x(x-1)+\mu_{1} y+\mu_{2} x y
\end{array}\right.
$$

and, among other results, he proves the following theorem:

Theorem 3.3 ([13]). There exists a unique analytic function $h\left(\mu_{2}\right)$ defined for all $\mu_{2} \in \mathbb{R}$ that satisfies $h^{\prime}(0)=-1 / 7, h\left(-\mu_{2}\right)=-h\left(\mu_{2}\right)$, and $\max \left(-1,-\mu_{2}^{2}\right)<$ $\mu_{2} h\left(\mu_{2}\right)<0$ for all $\mu_{2} \neq 0$ such that

(a) System (18) has a unique, hyperbolic limit cycle if and only if $\mu_{1} \mu_{2}<0$ and $0<\left|\mu_{1}\right|<\left|h\left(\mu_{2}\right)\right|$; the limit cycle is stable if $\mu_{1}>0$, and unstable if $\mu_{1}<0$;

(b) For $\mu_{2} \neq 0$, system (18) has a fine focus of multiplicity one at the origin if and only if $\mu_{1}=0$; for $\mu_{2}<0$, a unique, stable, limit cycle is generated in a supercritical Hopf bifurcation at the origin of (18) at the bifurcation value $\mu_{1}=0$, and it expands monotonically with increasing $\mu_{1}$ until it intersects the saddle at $(1,0)$ and forms a homoclinic loop at the bifurcation value $\mu_{1}=h\left(\mu_{2}\right)$;

(c) System (18) has a homoclinic loop at the saddle $(1,0)$ if and only if $\mu_{1}=h\left(\mu_{2}\right)$; the separatrix cycle is hyperbolic if and only if $\mu_{2} \neq 0$; it is stable (unstable) on its interior for $\mu_{2}<0\left(\mu_{2}>0\right)$. 
Let us relate the results of the above theorem with our results. We need the following simple lemma.

Lemma 3.4. When $\mu_{2} \neq 0$ the change of variables and time

$$
u=\frac{\mu_{2}^{2}}{2}(2 x-1), \quad v=\mu_{2}^{3} y, \quad \tau=\frac{t}{\mu_{2}}
$$

transforms system (18) into

$$
\left\{\begin{array}{l}
u^{\prime}=\frac{d u}{d \tau}=v \\
v^{\prime}=\frac{d v}{d \tau}=-\frac{\mu_{2}^{4}}{4}+\frac{\mu_{2}\left(2 \mu_{1}+\mu_{2}\right)}{2} v+u^{2}+u v .
\end{array}\right.
$$

As a consequence of the above lemma and Theorems 3.1, 1.2.(ii) and 3.3 we obtain:

Corollary 3.5. (i) The following relation holds

$$
\begin{aligned}
h\left(\mu_{2}\right) & =-\frac{\mu_{2}}{2}+\frac{b^{*}\left(\frac{\mu_{2}^{4}}{4}\right)}{\mu_{2}} \\
& =-\frac{1}{7} \mu_{2}+\frac{18}{2401} \mu_{2}^{3}-\frac{3753}{45294865} \mu_{2}^{5}-\frac{294120207}{22216678333850} \mu_{2}^{7}+O\left(\mu_{2}^{9}\right),
\end{aligned}
$$

where the functions $b^{*}$ and $h$ are the ones defined in Theorems 3.1 and 3.3, respectively. $H$.

(ii) The function $b^{*}(n)$ is given by $b^{*}(n)=H(\sqrt{n})$, for some analytic function

Proof. By using Lemma 3.4 we get that the relations between the variables in system (18) and the ones of system (2) are

$$
n=\frac{\mu_{2}^{4}}{4}, \quad b=\frac{\mu_{2}\left(2 \mu_{1}+\mu_{2}\right)}{2} .
$$

By using them we easily obtain the proof, because the relation $b=b^{*}(n)$ writes as

$$
\frac{\mu_{2}\left(2 \mu_{1}+\mu_{2}\right)}{2}=b^{*}\left(\frac{\mu_{2}^{4}}{4}\right)
$$

which immediately leads to (19). From this relation and the expression of $b=b^{*}(n)$ given in Theorem 1.2.(ii), the expansion (20) follows. Item (ii) is a consequence of (19), Theorem 3.3 and the fact that $h$ is an odd function.

We end this section with several remarks.

Remark 3.6. (i) Expression (20) improves the local knowledge of the function $h$ given in Theorem 3.3. The proof of [13] that $h^{\prime}(0)=-1 / 7$, is equivalent to the classical one which appears in [8] and gives the term $\frac{5}{7} \sqrt{n}$ in expression (13). Our approach goes much further and it is completely different.

(ii) By using Theorem 1.2.(i) we obtain the following global result: for $\left|\mu_{2}\right| \leq$ $10^{-1 / 4} \simeq 0.562$

$$
\left|-\frac{1}{7} \mu_{2}+\frac{18}{2401} \mu_{2}^{3}-h\left(\mu_{2}\right)\right| \leq \frac{11}{2^{9 / 2}}\left|\mu_{2}\right|^{4} .
$$


Notice that this inequality proves that for $\mu_{2} \in\left(-10^{-1 / 4}, 0\right)$,

$$
h\left(\mu_{2}\right)<-\frac{1}{7} \mu_{2}
$$

This result is coherent with the conjecture made in [13] that affirms that the above inequality holds for all $\mu_{2}<0$.

(iii) The inequalities of Lemma 3.2 translated to system (18) read as

$$
\max \left(-1,-\mu_{2}^{2}\right)<\mu_{2} h\left(\mu_{2}\right)<0 .
$$

This information is already contained in Theorem 3.3, but our proof is different to the one given in [13].

(iv) In [13] there is another conjecture that says that $\mu_{2} h\left(\mu_{2}\right)+1=O\left(1 / \mu_{2}\right)$ as $\mu_{2} \rightarrow-\infty$. In the parameters of the Bogdanov-Takens system (2) it reads as

$$
b^{*}(n)=\sqrt{n}-1+O\left(n^{-\frac{1}{4}}\right) \quad \text { as } n \rightarrow \infty .
$$

(v) The case $\mu_{2}=0$ includes new systems which are not contained in the expression of the Bogdanov-Takens system written as in (2). For instance the case $\mu_{1}=\mu_{2}=0$ corresponds to a Hamiltonian system with a center at the origin.

3.6. A final application. In [9] it is proved the following result:

Theorem 3.7 ([9]). The system

$$
\left\{\begin{array}{l}
\dot{x}=y, \\
\dot{y}=\beta y-\alpha x^{2}+\alpha^{2} x-x y, \text { with } \quad \alpha<0,
\end{array}\right.
$$

has a limit cycle if and only if $\gamma<\beta / \alpha<1$, where $\gamma$ is a positive constant.

This constant $\gamma$ is computed numerically in that paper as $\gamma \simeq 0.864546$. We remark that for the values $\alpha$ and $\beta$ satisfying $\beta / \alpha=\gamma$, the system (21) has a homoclinic loop through the origin. We will improve the results of that paper. We start by proving the following lemma.

Lemma 3.8. The constant $\gamma$ defined above is

$$
\gamma=b^{*}(1 / 4)+1 / 2
$$

where $b^{*}(n)$ is the function introduced in Theorem 3.1.

Proof. By applying the change of variables

$$
u=\frac{1}{2}-\frac{x}{\alpha}, \quad v=-\frac{y}{\alpha^{2}}, \quad \tau=\alpha t,
$$

to the system (21) we obtain

$$
\left\{\begin{array}{l}
u^{\prime}=\frac{d u}{d \tau}=v \\
v^{\prime}=\frac{d v}{d \tau}=-\frac{1}{4}+\left(\frac{\beta}{\alpha}-\frac{1}{2}\right) v+u^{2}+u v
\end{array}\right.
$$

Hence, the correspondence between the parameters of system (21) and the ones of the Bogdanov-Takens system (2) is

$$
n=1 / 4 \quad \text { and } \quad b=\beta / \alpha-1 / 2 .
$$

From these relations the lemma follows. 
As we have already explained at the end of Subsection 3.3 we have computed $b_{\text {num }}^{*}(n)$ at several values of $n$, obtaining in particular that

$$
\gamma \simeq b_{\text {num }}^{*}(1 / 4)+1 / 2=0.864545247421507=: \gamma_{\text {num }} .
$$

Notice that only the first five significative digits agree with that computed in [9], 0.864546. As we will prove in Theorem 3.10, at least eight of the significative digits of $\gamma_{\text {num }}$ are correct and $\left|\gamma-\gamma_{\text {num }}\right|<1.2 \times 10^{-9}$.

Let us call $\gamma_{k}:=b_{k}^{*}(1 / 4)+1 / 2$, where $b_{k}^{*}(n)$ is the function obtained by adding the first $k$ terms of the asymptotic expansion of $b^{*}(n)$ at the origin, see Theorem 1.2.(ii) and expression (17). For instance

$$
b_{1}^{*}(n)=\frac{5}{7} n^{1 / 2}, \quad b_{2}^{*}(n)=\frac{5}{7} n^{1 / 2}+\frac{72}{2401} n
$$

and so on. Although we have not proved neither the validity of these expressions until $n=1 / 4$ nor the validity of $b_{k}^{*}(1 / 4), k=5, \ldots, 11$ we can compute the values $b_{k}^{*}(1 / 4)+1 / 2$ and compare with the numerical approximation of $\gamma$. We obtain

$$
\begin{aligned}
& \gamma_{1}=\frac{6}{7} \simeq 0.857142857143, \quad\left|\gamma_{1}-\gamma_{\text {num }}\right| \leq 7.5 \times 10^{-3}, \\
& \gamma_{2}=\frac{2076}{2401} \simeq 0.864639733444, \quad\left|\gamma_{2}-\gamma_{\text {num }}\right| \leq 9.5 \times 10^{-5}, \\
& \gamma_{3}=\frac{39159987}{45294865} \simeq 0.864556876370, \quad\left|\gamma_{3}-\gamma_{\text {num }}\right| \leq 1.2 \times 10^{-5}, \\
& \gamma_{4}=\frac{19207287903423}{22216678333850} \simeq 0.864543637658, \quad\left|\gamma_{4}-\gamma_{\text {num }}\right| \leq 1.7 \times 10^{-6}, \\
& \gamma_{5}=\frac{9421002777077246787}{10897058555970086500} \simeq 0.864545485251, \quad\left|\gamma_{5}-\gamma_{\text {num }}\right| \leq 2.4 \times 10^{-7}, \\
& \gamma_{6}=\frac{11222200726046133491344191}{12980467181286007337935000} \simeq 0.864545210070, \quad\left|\gamma_{6}-\gamma_{\text {num }}\right| \leq 3.8 \times 10^{-8}, \\
& \gamma_{7}=\frac{935744176562 \cdots 60335616104987}{108235418911 \cdots 66123548550000} \simeq 0.864545253274, \quad\left|\gamma_{7}-\gamma_{\text {num }}\right| \leq 5.9 \times 10^{-9} \text {, } \\
& \gamma_{8}=\frac{2075476618505 \cdots 38798344748153}{2400657023927 \cdots 64252511900000} \simeq 0.864545246497, \quad\left|\gamma_{8}-\gamma_{\text {num }}\right| \leq 9.2 \times 10^{-10} \text {, } \\
& \gamma_{9}=\frac{7562725921574 \cdots 99070483500549}{8747634600776 \cdots 79842499000000} \simeq 0.864545247569, \quad\left|\gamma_{9}-\gamma_{\text {num }}\right| \leq 1.5 \times 10^{-10} \text {, } \\
& \gamma_{10}=\frac{1377872021601 \cdots 10350610970071}{1593753508852 \cdots 40373950000000} \simeq 0.864545247398, \quad\left|\gamma_{10}-\gamma_{\text {num }}\right| \leq 2.4 \times 10^{-11} \text {, } \\
& \gamma_{11}=\frac{5020759255426 \cdots 85210508369767}{5807399057919 \cdots 86029500000000} \simeq 0.864545247425, \quad\left|\gamma_{11}-\gamma_{\text {num }}\right| \leq 3.9 \times 10^{-12} \text {. }
\end{aligned}
$$

Notice that the values $\gamma_{k}$ given above approach well to $\gamma_{\text {num }}$, and by using again the results of Theorem 3.10, also approach to $\gamma$.

In fact in the sequel we will see how the method introduced in this paper allows to give a concrete interval where the actual value of $\gamma$ lies.

Recall that our approach for obtaining information of whether a homoclinic connection appears passes trough the construction of the two polynomials

$$
\begin{aligned}
& C_{k}(x, y)=C_{2}(x, y)+\sum_{3 \leq i+j \leq k} c_{i, j}^{k} x^{i} y^{j}, \\
& D_{k}(x, y)=\frac{\partial C_{k}(x, y)}{\partial x} y+\frac{\partial C_{k}(x, y)}{\partial y}\left(2 m^{2} x+\left(b+m^{2}\right) y+x^{2}+x y\right),
\end{aligned}
$$

with $k \geq 3$, and their resultant. From a computational point of view it is much simpler the case where all the numbers implied in their obtention are rational and then $C_{k}(x, y)$ and $D_{k}(x, y)$ are in $\mathbb{Q}[x, y]$. It is easy to see that this happens when $b$ and the eigenvalues of the saddle point given in (7) are rational numbers. From now one we will particularize our study to case $n=1 / 4$, that is $m^{2}=1 / 2$, 
although clearly our approach can be adapted to all values of $n$ such that $\sqrt{n}$ is a rational number. In our case, the rationality conditions are reduced to

$$
b=\frac{p}{q} \quad \text { and } \quad\left(\frac{p}{q}+\frac{1}{2}\right)^{2}+4=\left(\frac{r}{s}\right)^{2}
$$

for some integer numbers $p, q, r$ and $s$. This is equivalent to find integer solutions $p, q$ and $t$ of the quadratic diophantine equation

$$
4 p^{2}+4 p q+17 q^{2}=t^{2} .
$$

It is well-known how to study this type of equations. More concretely, if $\left(p_{0}, q_{0}, t_{0}\right) \in$ $\mathbb{Z}^{3}$ is a particular solution of the diophantine equation

$$
A p^{2}+B p q+C q^{2}=D t^{2},
$$

where $A, B, C$ and $D$ are integers numbers, then

$$
\begin{aligned}
& p=p(u, v)=\left(A p_{0}+B q_{0}\right) u^{2}+2 C q_{0} u v-C p_{0} v^{2}, \\
& q=q(u, v)=-A q_{0} u^{2}+2 A p_{0} u v+\left(B p_{0}+C q_{0}\right) v^{2}, \\
& t=t(u, v)=t_{0}\left(A u^{2}+B u v+C v^{2}\right),
\end{aligned}
$$

for any $(u, v) \in \mathbb{Z}^{2}$, is also an integer solution of the diophantine equation, because it holds that

$$
\begin{aligned}
& A p(u, v)^{2}+B p(u, v) q(u, v)+C q(u, v)^{2}-D t(u, v)^{2}= \\
& \left(A u^{2}+B u v+C v^{2}\right)^{2}\left(A p_{0}^{2}+B p_{0} q_{0}+C q_{0}^{2}-D t_{0}^{2}\right) .
\end{aligned}
$$

For equation (24) it is clear that $\left(p_{0}, q_{0}, t_{0}\right)=(1,0,2)$ is a particular solution. By applying the above procedure we obtain the new solutions

$$
\begin{aligned}
& p=p(u, v)=4 u^{2}-17 v^{2}, \\
& q=q(u, v)=4 v(2 u+v), \\
& t=t(u, v)=2\left(4 u^{2}+4 u v+17 v^{2}\right) .
\end{aligned}
$$

From them we obtain the suitable candidates to perform our study. We consider, for $(u, v) \in \mathbb{Z}^{2}$, with $v(2 u+v) \neq 0$,

$$
b=g(u, v)=\frac{4 u^{2}-17 v^{2}}{4 v(2 u+v)} \in \mathbb{Q},
$$

for which the eigenvalues of the saddle point given in (7), $a_{1}^{ \pm}$, are

$$
a_{1}^{+}=\frac{2 u+v}{4 v} \in \mathbb{Q} \quad \text { and } \quad a_{1}^{-}=-\frac{4 v}{2 u+v} \in \mathbb{Q} .
$$

The above results will allow us to prove the following lemma.

Lemma 3.9. Fix $n=1 / 4$ and $b \in \mathbb{R}$. Then there are infinitely many sequences of rational numbers $\left\{b_{j}\right\}_{j \in \mathbb{N}}$ such that $\lim _{j \rightarrow \infty} b_{j}=b$ and the eigenvalues $a_{1}^{ \pm}$of the saddle point

$$
\frac{b_{j}+1 / 2 \pm \sqrt{\left(b_{j}+1 / 2\right)^{2}+4}}{2}
$$

are also rational numbers. Moreover these sequences can be explicitly obtained. 
Proof. Notice that, for $v \neq 0$, the map $g(u, v)$ given in $(25)$ can be written as

$$
b=g(u, v)=\frac{4\left(\frac{u}{v}\right)^{2}-17}{8\left(\frac{u}{v}\right)+4} .
$$

Then the first part of the proof follows by noticing that the graph of the map

$$
w \longmapsto G(w)=\frac{4 w^{2}-17}{8 w+4}, \quad w \in \mathbb{R}
$$

covers all the real line. To prove the second part it suffices to consider any sequence of rational numbers $\left\{w_{j}\right\}_{j \in \mathbb{N}}$ tending to one of the preimages for $G$ of $b$, say $G^{-1}(b)$. Then, clearly, it holds that for any $j \in \mathbb{N}$

$$
b_{j}:=G\left(w_{j}\right)=\frac{4 w_{j}^{2}-17}{8 w_{j}+4} \in \mathbb{Q}, \quad \frac{b_{j}+1 / 2 \pm \sqrt{\left(b_{j}+1 / 2\right)^{2}+4}}{2} \in \mathbb{Q}
$$

and $\lim _{j \rightarrow \infty} b_{j}=b$ as we wanted to prove.

Finally, we prove:

Theorem 3.10. Let $\gamma$ be the real number introduced in Theorem 3.7. Then

$$
\gamma \in\left(\frac{1127949288}{1304673517}, \frac{1223980221}{1415750330}\right) \simeq(0.864545247,0.8645452486) \text {. }
$$

Notice that the length of the interval given in the above theorem is smaller than $1.61 \times 10^{-9}$.

Proof. By using Lemma 3.8, $\gamma=b^{*}(1 / 4)+1 / 2$. So we will fix $n=1 / 4$ and we study $b^{*}:=b^{*}(1 / 4)$, for system (2). For the sake of simplicity we only give the full details of the range of values of $b^{*}$ that we obtain by applying our method with $k=3$, that is by using a cubic curve (23). The proof of the theorem follows by taking $k=6$.

The numerical approximation $(22)$ of $b^{*}$ gives an orientation for the actual value of $b^{*}$. After some trials we consider the values

$$
b_{3}^{\ell}:=G\left(\frac{19}{8}\right)=\frac{89}{368} \quad \text { and } \quad b_{3}^{u}:=G\left(\frac{8}{3}\right)=\frac{103}{228},
$$

where $G$ is the function given in $(26)$, and we prove that $b^{*} \in\left(b_{3}^{\ell}, b_{3}^{u}\right)$. Notice that $(89 / 368,103 / 228) \simeq(0.242,0.452)$.

We will apply the procedure introduced in Section 3 for $n=1 / 4$ and $b \in\left(b_{3}^{\ell}, b_{3}^{u}\right)$ for searching suitable cubic curves, both having an oval through the origin and being without contact with the flow of the system. Moreover we will prove that for one of the ovals the flow goes inwards and for the other one the flow goes outwards.

Fix one of the values, say $b=89 / 368$. Then we apply our procedure to determine $C_{3}(x, y)$, by imposing all the coincidence conditions between the cubic and the separatrix associated to the eigenvalue $a_{1}^{+}=23 / 16$. Recall that due to our choice of $b$ we can ensure that all our computations will be with polynomials in $\mathbb{Q}[x, y]$. We obtain

$$
\begin{aligned}
C_{3}^{\ell}(x, y)= & \frac{(16 x+23 y)(16 y-23 x)}{368}-\frac{30470974207443747849}{44286028769220680429} x^{3}-\frac{48084904789188461109}{88572057538441360858} x^{2} y \\
& +\frac{300486284549883520}{44286028769220680429} x y^{2}-\frac{78703862917780480}{132858086307662041287} y^{3} .
\end{aligned}
$$


We omit the explicit expression of $D_{3}^{\ell}(x, y) \in \mathbb{Q}[x, y]$, which has degree 4 . The resultant of $C_{3}^{\ell}(x, y)$ and $D_{3}^{\ell}(x, y)$, with respect to $y, \operatorname{Res}\left(C_{3}^{\ell}, D_{3}^{\ell}, y\right)$ is a polynomial of the form $x^{10}\left(A x^{2}+B x+C\right) \in \mathbb{Q}[x]$, for some huge rational numbers (numerators and denominators with more than 60 digits), and $A>0, B<0$ and $C>0$. Hence the resultant is positive in $\{(x, y): x<0\}$.

If we compute the resultant of $C_{3}^{\ell}(x, y)$ and $\partial C_{3}^{\ell}(x, y) / \partial y$ with respect to $y$ we obtain a polynomial of the form $x^{2} P_{4}(x)$, with $P_{4}(x) \in \mathbb{Q}[x]$. The roots of $P_{4}$ can be explicitly obtained (or located by using the Sturm sequences of $P_{4}$, in view of the application of our approach for $k>3$ ). Their approximate values are $-24.478,-9.855, x_{0} \simeq-1.454$ and 32.737 .

The following facts are not difficult to prove for the cubic $C_{3}^{\ell}(x, y)=0$ :

- Its only multiple point is the origin, which is a double point. On it, the curve has two smooth branches tangent to the lines $(16 x+23 y)(16 y-$ $23 x)=0$;

- The polynomial $C_{3}^{\ell}(0, y)$ has the 0 as a double root and a simple nonzero root;

- For each $x \in\left(x_{0}, 0\right)$ the polynomial $y \longmapsto C_{3}^{\ell}(x, y)$ has exactly three simple real roots;

- The polynomial $y \longmapsto C_{3}^{\ell}\left(x_{0}, y\right)$ has a simple root and a root of multiplicity two;

- For each $x \in\left[-2, x_{0}\right)$ the polynomial $y \longmapsto C_{3}^{\ell}(x, y)$ has exactly one simple real root.

The tool that we use to prove the first item is the computation of the resultants between $C_{3}^{\ell}(x, y), \partial C_{3}^{\ell}(x, y) / \partial x$ and $\partial C_{3}^{\ell}(x, y) / \partial y$. To prove the other ones we compute the Sturm sequences of the polynomials $C_{3}^{\ell}(x, y)$ considered as polynomials in $y$, with coefficients in the rational functions with numerators and denominators in $\mathbb{Q}[x]$. This can be done, except at finitely many points, given by the zeros of some polynomial of $x$ that appear during the process. In particular, we can see that studying the four polynomials $C_{3}^{\ell}(x, y)$ for $x \in\left\{-2, x_{0},-1,0\right\}$ we have all the information for $y \longmapsto C_{3}^{\ell}(x, y)$ and $x \in[-2,0]$.

The above list of properties prove that the curve $C_{3}^{\ell}(x, y)=0$ has a loop which starts and ends at the origin being tangent to the lines $(16 x+23 y)(16 y-23 x)=0$ and is contained in the strip $\left\{(x, y): x_{0} \leq x \leq 0\right\}$. Since we have proved that the resultant $\operatorname{Res}\left(C_{3}^{\ell}, D_{3}^{\ell}, y\right)$ does not vanish on the left hand plane $\{(x, y): x<0\}$ we know that the oval is without contact for the flow of the system. By studying the sign of $D_{3}^{\ell}$ on the loop we prove that the flow crosses it inwards. Hence we have proved that $b^{*}>b_{3}^{\ell}=89 / 368$.

Working similarly, for $b=103 / 228$ and $a_{1}=19 / 12$ we obtain the cubic

$$
\begin{aligned}
C_{3}^{u}(x, y)= & \frac{(12 x+19 y)(12 y-19 x)}{228}-\frac{42394240475656582327}{66085291294166321043} x^{3}-\frac{24891674002318104595}{44056860862777547362} x^{2} y \\
& +\frac{320066250082464600}{22028430431388773681} x y^{2}-\frac{37723016690931312}{22028430431388773681} y^{3}
\end{aligned}
$$

and we prove that $b^{*}<b_{3}^{u}=103 / 228$.

Before considering greater values of $k$ we want to comment that, also keeping $k=3$, but taking rational numbers with big numerators and denominators, we could improve a little bit the knowledge of the interval where $b^{*}$ lies. For instance we can prove that 


$$
b^{*} \in\left(G\left(\frac{951}{398}\right), G\left(\frac{29}{11}\right)\right)=\left(\frac{28898}{114425}, \frac{1307}{3036}\right) \simeq(0.2525,0.4305) .
$$

By applying our method for $k>3$ we obtain that

$$
\begin{array}{ll}
b^{*} \in\left(\frac{1300991}{3571092}, \frac{411011}{112574}\right) \simeq(0.364312,0.365103) & \text { for } \quad k=4, \\
b^{*} \in\left(\frac{357550843}{980814604}, \frac{67268863}{184528084}\right) \simeq(0.3645448,0.3645454) & \text { for } k=5, \\
b^{*} \in\left(\frac{951225059}{2609347034}, \frac{258052528}{707875165}\right) \simeq(0.364545247,0.3645452486) & \text { for } \quad k=6 .
\end{array}
$$

We notice that the theorem follows from the result when $k=6$.

All the computations for the six values of $b$ to be studied follow a similar procedure to the one described above. The main computational difficulties appear for proving that the algebraic curve of degree $k$ has a transversal oval passing through the origin due to the high degree and huge size of the coefficients of the polynomials involved. For all cases we prove for the algebraic curve $C_{k}(x, y)=0, C_{k}(x, y) \in$ $\mathbb{Q}[x, y]$, the following properties, which are similar to the ones given for $k=3$. In each of the six cases there is a different value, say $z_{0} \in(-2,-1)$, which is given as a zero of a polynomial with rational coefficients computed through a suitable resultant.

- The only multiple point is the origin, which is a double point. On it, the curve has two smooth branches tangent to two given lines with rational slopes;

- the polynomial $C_{k}(0, y)$ has the 0 as a double root and $k-2$ simple nonzero roots;

- for each $x \in\left(z_{0}, 0\right)$ the polynomial $y \longmapsto C_{k}(x, y)$ has exactly $k$ simple real roots;

- the polynomial $y \longmapsto C_{k}\left(z_{0}, y\right)$ has $k-2$ simple roots and a root of multiplicity two;

- for each $x \in\left[-2, z_{0}\right)$ the polynomial $y \longmapsto C_{k}(x, y)$ has exactly $k-2$ simple real roots.

\section{REFERENCES}

[1] A. A. Andronov, E. A. Leontovich, I. I. Gordon and A. G. Mă̌er, Theory of bifurcations of dynamic systems on a plane. Translated from the Russian. Halsted Press [A division of John Wiley \& Sons], New York-Toronto, Ont.; Israel Program for Scientific Translations, Jerusalem-London, 1973.

[2] B. Boisseau, P. Forgács, and H. Giacomini, An analytical approximation scheme to twopoint boundary value problems of ordinary differential equations. J. Phys. A 40 F215-F221, (2007).

[3] Shui-Nee Chow, Chengzhi Li and Duo Wang, Normal forms and bifurcation of planar vector fields. Cambridge University Press, Cambridge, 1994.

[4] B. Coll, A. Gasull and J. Llibre, Quadratic systems with a unique finite rest point. Publ. Mat. 32, 199-259, (1988).

[5] W. A. Coppel, A survey of quadratic systems. J. Differential Equations 2, 293-304, (1966).

[6] G. F. D. Duff, Limit-cycles and rotated vector fields. Ann. of Math. 67, 15-31, (1953).

[7] F. Dumortier and P. Fiddelaers, Quadratic models for generic local 3-parameter bifurcations on the plane. Trans. Amer. Math. Soc. 326, 101-126, (1991). 
[8] J. Guckenheimer and P. Holmes, Nonlinear oscillations, dynamical systems, and bifurcations of vector fields. Applied Mathematical Sciences, 42. Springer-Verlag, New York, 1983.

[9] Gil-Jun Han, Bifurcation analysis on an unfolding of the Takens-Bogdanov singularity. J. Korean Math. Soc. 36, 459-467 (1999).

[10] Chengzhi Li, C. Rousseau and Xian Wang, Simple proof for the unicity of the limit cycle in the Bogdanov-Takens system. Canad. Math. Bull. 33, 84-92, (1990).

[11] F. Dumortier, J. Llibre and J. C. Artés, Qualitative theory of planar differential systems. Universitext. Springer-Verlag, Berlin, 2006.

[12] L. M. Perko, Rotated vector fields and the global behavior of limit cycles for a class of quadratic systems in the plane. J. Differential Equations 18, 63-86, (1975).

[13] L. M. Perko, A global analysis of the Bogdanov-Takens system. SIAM J. Appl. Math. 52, 1172-1192, (1992).

[14] L. M. Perko, Differential equations and dynamical systems. Third ed., Texts in Applied Mathematics, vol. 7, Springer-Verlag, New York, 2001.

[15] I. G. Rozet, The closing of the separatrices of a certain first order differential equation (Russian) Differencial'nye Uravnenija 7, 2007-2012, (1971). Translated to English in Differential Equations 7, 1517-1521, (1971).

Dept. de Matemàtiques. Universitat Autònoma de Barcelona. Edifici C. 08193 Bellaterra, BArcelona. Spain

E-mail address: gasull@mat.uab.cat

Laboratoire de Mathématiques et Physique Théorique. UMR 6083-CNRS- FaCulté des Sciences et Techniques. Université de Tours. 37200 Tours. France

E-mail address: Hector.Giacomini@lmpt.univ-tours.fr

Dept. de Matemàtiques, Universitat Autònoma de Barcelona, Edifici C. 08193 Bellaterra, Barcelona. Spain

E-mail address: torre@mat.uab.cat 